\title{
In vitro Antifungal Activity of a Novel Allylamine Antifungal Nanoemulsion Gel
}

\author{
Karri VVSR ${ }^{1 *}$, Raman SK ${ }^{1}$, Kuppusamy $\mathbf{G}^{1}$, Sanapalli BKR ${ }^{1}$, Wadhwani $\mathbf{A}^{2}$, Patel $\mathbf{V}^{2}$ and Malayandi $\mathbf{R}^{3}$ \\ ${ }^{1}$ Department of Pharmaceutics, JSS College of Pharmacy, Ootacamund, JSS Academy of Higher Education and Research, Mysuru, India \\ ${ }^{2}$ Department of Pharmaceutical Biotechnology, SS College of Pharmacy, Ootacamund, JSS Academy of Higher Education and Research, Mysuru, India \\ ${ }^{3}$ Pharmacokinetic Research and Development, Sun Pharmaceutical Industries Ltd, Baroda, India
}

*Corresponding author: Veera Venkata Satyanarayana Reddy Karri, Department of Pharmaceutics, JSS Academy of Higher Education and Research, Mysuru, India, Tel: +91-821-2548416 / 400; E-mail: ksnreddy87@gmail.com

Received date: February 7, 2018; Accepted date: February 19, 2018; Published date: February 26, 2018

Copyright: ( 2018 Karri VVSR, et al. This is an open-access article distributed under the terms of the Creative Commons Attribution License, which permits unrestricted use, distribution, and reproduction in any medium, provided the original author and source are credited.

\begin{abstract}
Terbinafine $\mathrm{HCl}(\mathrm{TBH})$ is a broad spectrum antifungal agent used against dimorphic fungi, yeast, molds, dematiaceous fungi and dermatophytes. However, the poor solubility of this potential molecule renders it efficacy limited. So, the combinations of oral and topical doses are prescribed. Hence, the aim of the current study was to increase the solubility of TBH by incorporating into Nanoemulsion gel (NEG) and to investigate it's in vitro antifungal activity using Candida albicans as a fungal inoculum. The TBH nanoemulsion was prepared using high speed homogenization technique and evaluated for its micromeritcs. The particles results revealed that acceptable globule size of $11.47 \mathrm{~nm}$ with a PDI of 0.556 . The obtained nanoemulsion was converted in to NEG and evaluated for in vitro permeation and in vitro antifungal activity. The permeation studies reveled that NEG $(76.23 \pm 2.81 \%)$ has higher permeation, when compared to marketed cream (MC $29.37 \pm 1.82 \%$ ). In vitro Antifungal studies showed that the prepared NEG $(0.66 \pm 0.17 \mathrm{~mm})$ has high zone of inhibition $(p<0.01)$ almost to that of pure drug solution $(0.91 \pm 0.07$ $\mathrm{mm})$ compared to MC $(0.13 \pm 0.05 \mathrm{~mm})$. Hence, the formulated NEG of TBH may best suitable for delivery of poorly soluble drugs like $\mathrm{TBH}$, which are intended for topical antifungal application.
\end{abstract}

Keywords: Nanoemulsion; Antifungal activity; Allylamine; Terbinafine $\mathrm{HCl}$

\section{Introduction}

Terbinafine $\mathrm{HCl}(\mathrm{TBH})$ is an orally administered, topically viable allylamine antifungal agent [1]. TBH possesses broad spectrum in vitro potential against dimorphic fungi, yeast, molds, dematiaceous fungi and dermatophytes. TBH is active in vitro against a wide range of dermatophytes, dimorphic fungi, molds and yeasts. However, previous studies suggest that the TBH had little activity against Candida albicans (C. albicans) [2]. Candida species are considered as the most important fungal human pathogens, causing a variety of infections, ranging from superficial, cutaneous-mucosal to deep-seated infections [3]. Candidiasis or yeast infection is the most common skin infection caused by the $C$. albicans. Among the various types of candidiasis, cutaneous candidiasis skin fungal infections will most often reoccur and is rarely cured [4]. TBH is the most successful compound in the category of antifungals, when compared to other antifungals viz.,butenafine, ketoconazole and itraconazole etc. [5]. However, the poor solubility of this potential molecule renders it efficacy limited [6]. Hence, the combinations of oral and topical doses are prescribed. The development of nanoparticles $(1-100 \mathrm{~nm})$ for topical application has been emerging in the past decadeand offer potential applications in topical drug delivery [7]. These systems increase the drug permeability of poorly soluble drugs and provide enhanced drug availability at the targeted site. They also create a drug depot at the site of action $[8,9]$. The controlled release properties of nanoparticles facilitate pulsatile release of drug molecules minimizing the frequent and total number of dosages, therebyoffering increased patient compliance. Potent bactericidal activity against gram positive and negative bacteria has been seen in nanoparticles encompassing antibiotics [10,11], which can be extrapolated for possible antifungal efficacy too. Silver nanoparticles, an example of antimicrobial NPs have been recently developed [12,13], to counteract the constantly evolving bacterial resistance to regular antibiotics. Another advantage of using NPs as a carrier for antifungal and antibiotic drugs lies in the fact that NPs exhibit bactericidal action at low concentrations (units of $\mathrm{mgL}^{-1}$ ) [14], thereby reducing the toxic burden of these drugs on human cells. In the present study, an attempt was made to prepare a nano particulated drug delivery system for TBH and its antifungal efficacy was evaluated by in vitro microbial assay using C.albicansas fungal inoculum.

\section{Materials and Methods}

TBH was purchased from Hetero labs, Hyderabad, India. Liquid paraffin was purchased from Unicorn (Chennai, India). Polysorbate 80 was purchased from N.O.F Corporation (Mumbai, India). Milli Q water was prepared by Milli-Q plus 185 purification system (Bangalore, India), which was used throughout the study. Marketed cream (MC) containing $1 \%$ of TBH dispersed in cream base was used as standard for the all the studies.

\section{Development of calibration curve by HPLC}

Calibration curve of TBH was performed by Liquid chromatographic analysis as previously described [15]. Briefly, HPLC system (Shimadzu, Japan) comprising LC-20AD solvent pump and a SPD-M20A PDA detectorwere used in this study. The LC solutions (ver. 1.11) software was used for data analysis and processing. Chromatographic separation was performed onLuna $\mathrm{C}_{18}$ (Phenomenex, California, USA) $(250 \times 4.6 \mathrm{~mm})$ column using a mobile 
phase comprising $25 \mathrm{mM}$ potassium dihydrogen orthophosphate buffer at $\mathrm{pH} 4.0$ adjusted with orthophosphoric acid and methanol in the ratio of 20:80 at $222 \mathrm{~nm}$. The mobile phase was delivered at $1 \mathrm{~mL}$ $\min ^{-1}$.

\section{Preparation of nanoemulsion gel}

TBH nanoemulsion was prepared by the high speed homogenization method (PT-3100D, Kinematica, Schweiz) reported elsewhere [14]. The nanoemulsion contains two phases i.e., aqueous phase and oily phase. Sodium acetate, disodium edate, glycerin and polysorbate 80 were dissolved in purified water (continuous phase). TBH was dispersed in liquid paraffin (dispersed phase). The dispersed phase was added to continuous phase by high speed homogenization. The rpm was maintained in the range of 4000-5000 for $1 \mathrm{~h}$. After homogenization, the resulting nanoemulsion was incorporated into carbomer 970 gel base (1.5\%). The carbomer 970 gelbasewas prepared in advance by addition of $1.5 \mathrm{~g}$ of carbomer 970 into water with continuous stirring. Subsequently, the $\mathrm{pH}$ of prepared nanoemulsion gel (NEG) of TBH was adjusted to 5.5-6.0 using $2 \mathrm{~N} \mathrm{NaOH}$. The detailed composition of NEG is described in Table 1.

\begin{tabular}{|l|l|l|}
\hline Formula & Category/Role & Concentration range (\%Wt/Wt) \\
\hline TFH & Active Pharmaceutical Ingredient & $1 \%$ \\
\hline Liquid paraffin & Carrier & $5 \%$ \\
\hline Polysorbate 80 & o/w Emulsifier & $2 \%$ \\
\hline Glycerin & Humectant & $1 \%$ \\
\hline Sodium acetate & Buffering agent & $0.20 \%$ \\
\hline Edetate disodium & Anti-Oxidant & $0.02 \%$ \\
\hline Benzyl alcohol & Preservative & $2 \%$ \\
\hline TFH & Active Pharmaceutical Ingredient & $1 \%$ \\
\hline
\end{tabular}

Table 1: Composition of TBH-NEG formulation.

\section{Drug content determination}

Each one gram of NEG was dissolved in methanol. The solution was sonicated for $2 \mathrm{~h}$ using Ultrasonicator (Equitron, Mumbai, India). The resulting suspension was centrifuged at 25,000 rpm (Model-R8C, Remi-Centrifuge, India) for $1 \mathrm{~h}$ and the resulting supernatant was filtered through $0.45 \mu \mathrm{m}$ filter to obtain clear solution. The drug content was estimated by HPLC as described under HPL analysis.

\section{Characterization}

Size and zeta potential: Dynamic light scattering technique was employed to measure mean droplet size and poly dispersity index of nanoemulsion using Malvern Zetasizer (Malvern, UK). The globule size and polydispersity index of the emulsion was assessedafter each cycle of high speed homogenization.

Transmission electron microscopic (TEM) analysis: To study the external morphology by TEM (100s, JEOL Ltd, Japan), a drop of nanoemulsion was placed on the copper grids and dried over night to remove the moisture completely. The analysis was performed at $200 \mathrm{kV}$.

Viscosity: The viscosity of NEG was determined by using Brookfield viscometer DV-II+ model having a T-bar spindle in combination with helical path.

In vitro permeation studies [16]: In vitro permeation studies of NEG and MC were carried in Franz diffusion cells with a diffusion area of $3.8 \mathrm{~cm}^{2}$. The receptor compartment was filled with $15 \mathrm{~mL}$ of phosphate buffer ( $\mathrm{pH}$ 7.4) and stirred continuously using magnetic stirrer at $300 \mathrm{rpm}$ [17]. Excised pork ear skin was fixed between donor and receptor compartment with the stratum corneum positioned towards the donor compartment [18]. $200 \mathrm{mg}$ of NEG and MC was placed separately on the skin. $1 \mathrm{~mL}$ of sample was withdrawn from each cell at predetermined intervals of $0,0.25,0.5,0.75,1,2,4,6$ and 9 hours and replaced with equal volume of buffer media [19]. At the end of study, the amount of permeated $\mathrm{TBH}$, retained on skin and within the skin was determined by HPLC analysis.

Microbiological assay: The microbiological assay of NEG and MC was carried out on C.albicans species using cup plate method. Petridishes containing $20 \mathrm{~mL}$ of medium (Sabouraud dextrose agar) were seeded with $100 \mu \mathrm{L}$ of the fungal inoculum (C.albicans). Consequently, each well in $2 \mathrm{~cm}$ diameter was cut out of the agar. $2 \mathrm{~g}$ of NEG (1\%) formulation and marketed cream (MC, 1\%) were placed into each well. For positive control, methanolic solution of TBH (1\%) equivalent to 2 $\mathrm{g}$ of formulation was used. Plates of $C$. albicans were incubated at $25^{\circ} \mathrm{C}$ for 2 days. After $48 \mathrm{~h}$, the zone of growth inhibition were observed and measured $[1,20]$. Statistical analysis using one-way ANOVA with Tukey's post-hoc test was performe dusing Graph Pad Prism software, version 6.0 (Graphpad Inc., USA).

\section{Results}

\section{Development of calibration curve}

Development of the calibration curve was performed to find out the linearity between concentration of the drug in the solution and its absorbance. The obtained data revealed that linearity was observed in the concentration range of $10-500 \mu \mathrm{g} / \mathrm{ml}$. The correlation coefficient obtained was found to be 0.999 with a slope $10702 \mathrm{x}$ and $\mathrm{y}$ intercept value of 46723 as shown in Figure 1. 


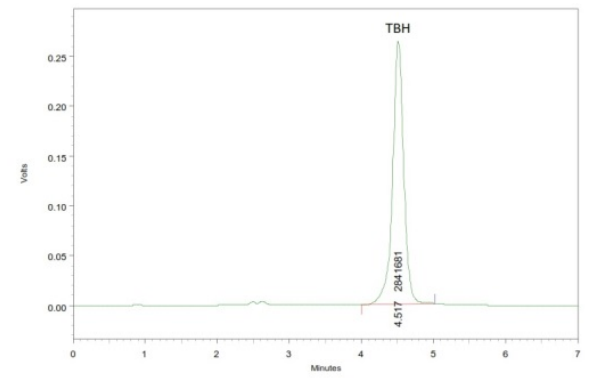

Figure 1: Chromatogram of terbinafine $\mathrm{HCl}$ in nanoemulsion gel formulation.

\section{Preparation and characterization of nanoemulsion}

Nanoemulsion was prepared using HSH with different paraments as described above. The nanoemulsion obtained is having a globule size of $11.47 \mathrm{~nm}$ with a poly dispersity index of 0.556 as shown in the Figure 2. Nanoemulsion was converted into nanoemulsion gel found to be homogenous without any difference in color intensity, separation of phase, aggregates, with smooth feel and non-greasy for ease of application with a $\mathrm{pH}$ of 5.8. $\mathrm{pH}$ was considered as the acceptable range as $\mathrm{pH}$ of the skin was 5.5 almost near which won't cause any irritation upon application on skin. The drug content of nanoemulsion gel using plotted calibration curve was found to be $98.09 \%$.

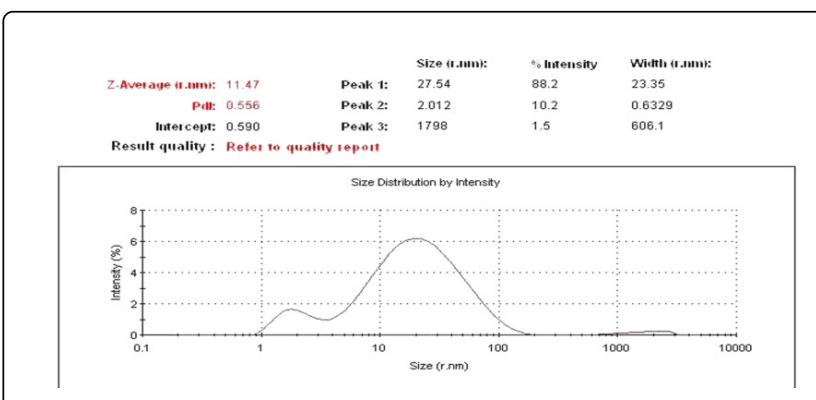

Figure 2: Size and polydispersityindex report of nanoemulsion.

\section{TEM analysis}

External morphological studies (TEM) revealed that particles in the nanoemulsion appeared as numerous, scattered, spherical dark stained circles possessing a size of $<20 \mathrm{~nm}$ as shown in Figure 3. The size of the nanoparticles observed by TEM correlated well the particle size measured by zeta sizer.

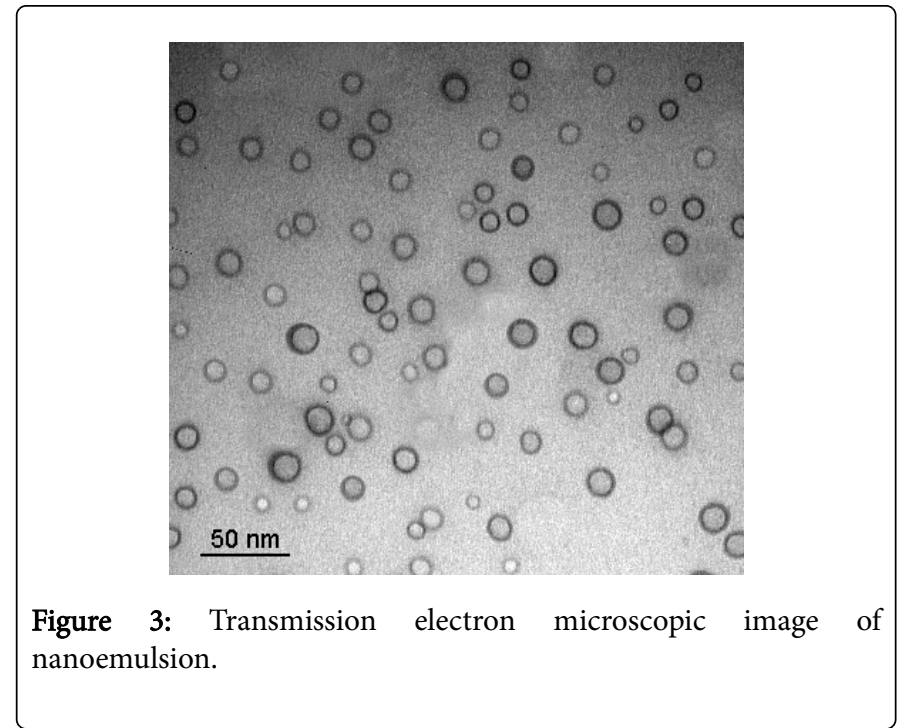

\section{Viscosity}

The viscosity of the formulation helps to show good spreadability ensure that a suitable dose is applied or delivered to the site of target. Viscosity of NEG was found to be in the range of 3,500-4,300 cps, while that of MC was found to be in the range of 5,200-6,000 cps. The viscosity is inversely proportional to efficacy, less the viscous more the spreadability which increases the drug delivery topically. On this basis it was conclude that NEG could be more efficient than MC.

\section{In vitro permeation study}

The percentage cumulative release of TBH permeated from NEG and MC until 24h is shown in Figure 4. The percentage of TBH permeated (receptor chamber), retained in the skin and on the skin (donor chamber) at the end of the study is presented in Figure 5.The total amount of TBH permeated wasobserved to be high with NEG (76 $\pm 0.9 \%)$ than MC $(29 \pm 0.8 \%)$. The MC had retained a largeamount of drug $(57.2 \pm 3.1 \%)$ on the skin.

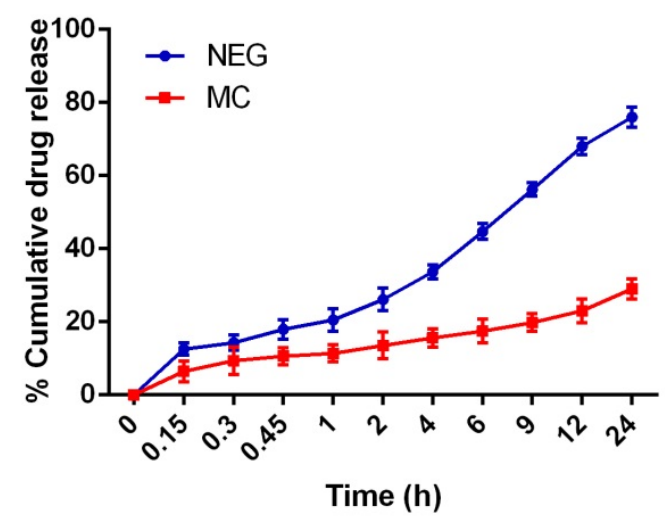

Figure 4: Permeation profiles of tested formulations (mean $\pm S D$, $\mathrm{n}=3)$. 


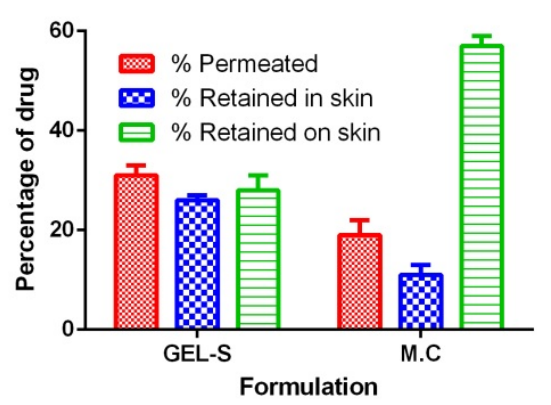

Figure 5: TBH in different compartments of skin after the in vitro permeation study (mean $\pm S D, n=3$ ).

\section{In vitro microbial assay}

The microbial assay for zone of inhibition was performed on fungal strain C.albicans. The maximum zone of inhibition was found to be $0.66 \pm 0.17 \mathrm{~mm}$ for NEG in comparison with MC $0.13 \pm 0.05 \mathrm{~mm}$ as shown in Figure 6. These results suggest that NEG could be more efficient than MC. This was attributed to greater penetration of TBH present in the size of nano in nanoemulsion.

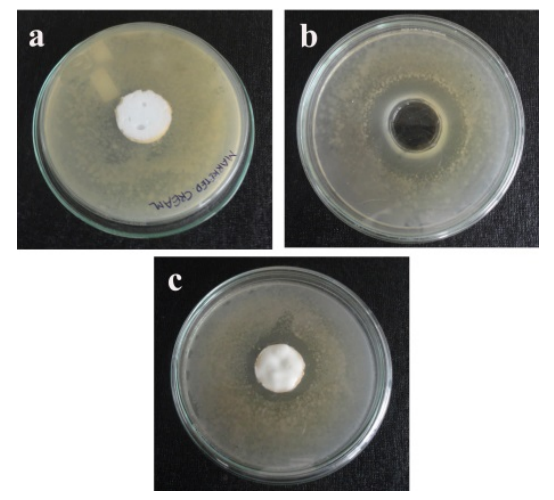

Figure 6: Plates showingzone of inhibition of (a) MC (b) pure drug solution (c) NEG.

\section{Discussion}

The prepared NEG was found to be homogenous and did not contain aggregated particles. The $\mathrm{pH}$ of NEG was found to be 5.8, which is nearly to the $\mathrm{pH}$ of normal skin ( $\mathrm{pH}-5.5)$ that ensures it will not produce any local irritation on skin. Extremes of $\mathrm{pH}$ on acidic and basic side can always cause irritation with redness at the site of application [21]. Topical application of particles requires less than 20 $\mathrm{nm}$ size [7] for high concentration of drug to penetrate the skin effectively into the stratum corneum. Hence, the obtained globule size $(11.47 \mathrm{~nm})$ was found to exist within the acceptable range for topical application i.e., $<20 \mathrm{~nm}$. The viscosity of prepared NEG was comparatively less than MC, since gels have lesser viscosity compared to creams. As viscosity is inversely proportional to spreadability, with the increase in viscosity, the spreadability and efficacy of the formulation is expected to decrease. Gelshave less viscosity with highest spreadability. This characteristic feature of gels helps in ensuring greater contact of drug with skin and has higher efficacy rates. Therefore, NEG is expected to have higher efficacy compared to MC. In vitro permeation studies revealed that the prepared NEG showed high penetration and retention in the skin compared to MC; this may be attributed to the lower globule size and lesser viscosity of NEG. Low size globules or particles have greater penetrability towards the stratum corneum and functionally create a large drug depot in the skin. The lipophilic nature of prepared nanoemulsions will have greater retention time in epidermis (lipophilic) and have more chances of sustained release patterns [22]. MC has less permeation, which may be attributed to its slower release (high viscosity) and poor permeation $[23,24]$. In the microbial assay, one-way ANOVA followed by Tukey'smultiple comparison test revealed NEG to be significantly effective $(\mathrm{p}<0.01)$ compared to $\mathrm{MC}$ in the zone of inhibition, which may be due to the high lipophilic nature of carrier (liquid paraffin), present as an $\mathrm{o} / \mathrm{w}$ nanoemulsion in NEG. No significant differences were observed between NEG and pure drug solution as shown in Figure 7. Hence, in the current study NEG is highly lipophilic compared to MC. As fungal strains have an innate specificity towards lipophilic compounds, the formulated NEG with distinct lipophilic characteristics is readily taken up by them, thereby resultingin high zone of inhibition.

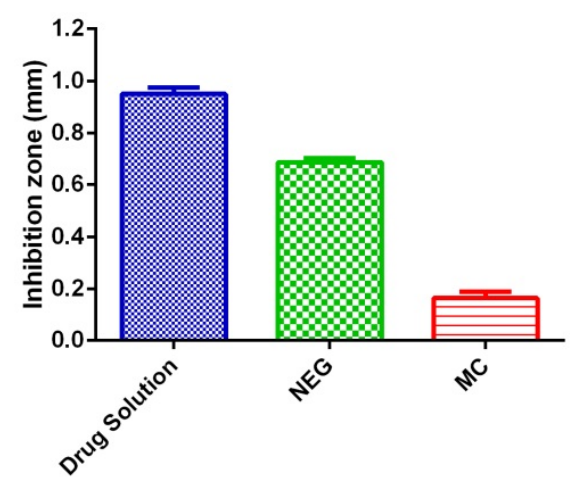

Figure 7: Comparative data of zone of inhibition.

\section{Conclusion}

The present $\mathrm{TBH}$ is available in market in different dosage forms like tablets, creams, gels, and sprays. But each having its own disadvantages like tablets with poor bioavailabity and should not be given for hepatic and cardiac patients, in case of creams and gels they are having poor penetration, hence require long term of therapy for curing and to minimize the relapse rates. Usually a combination of topical and oral antifungal is preferable with a minimum 14 days of therapy which increases the cost of therapy and decreases the patient compliance. Hence in the present study a novel dosage form for TBH is designed to increase its penetrability and reduce the treatment time there by increasing the patient compliance and reducing the cost of therapy. Accordingly the prepared NEG has overcome the problems associated with these poorly soluble drugs by enhancing the drug penetration and showing a maximum antifungal activity in vitro as that of drug. To conclude, the present study confirms that NEG of TBH 
Citation: Karri VVSR, Raman SK, Kuppusamy G, Sanapalli BKR, Wadhwani A, et al. (2018) In vitro Antifungal Activity of a Novel Allylamine Antifungal Nanoemulsion Gel. J Nanosci Curr Res 3: 119. doi:10.4172/2572-0813.1000119

Page 5 of 5

may be the best one for delivery of poorly soluble drugs like $\mathrm{TBH}$, which are intended for topical antifungal application.

\section{Future Perspective}

Future studies should be done on large number of animals and humans to evaluate the safety and efficacy of the prepared TBH-NEG. Since, these NEG technologies have greater scale up capabilities compared to other nano drug delivery systems, feasibility of product transfer from research and development to large scale has to be determined with the help of technology transfer.

\section{Conflict of Interest}

None declared.

\section{Note}

The content of thisarticle is the subject of Indian Patent Application number 1186/CHE/2013 dated 20/03/2013 titled "Composition of Terbinafine $\mathrm{HCl}$ topical nanoemulsion gel for treating fungal diseases" by the Author.

\section{References}

1. Cardoso SG, Schapoval EES (1999) High-performance liquid chromatographic assay of terbinafine hydrochloride in tablets and creams. J Pharm Biomed Anal 19: 809-812.

2. Ryder NS, Wagner S, Leitner I (1998) In vitro activities of terbinafine against cutaneous isolates of candida albicans and other pathogenic yeasts. Antimicrob Agents Chemother 42: 1057-1061.

3. Segal E (2005) Candida, still number one - what do we know and where are we going from there? Mycoses 48: 3-11.

4. Gupta M, Vyas SP (2012) Development, characterization and in vivo assessment of effective lipidic nanoparticles for dermal delivery of fluconazole against cutaneous candidiasis. Chem Phys Lipids 165: 454-461.

5. Volk B, Tiu A, St Anna L (2013) Clinical Inquiry: which oral antifungal works best for toenail onychomycosis? J Fam Pract 62: 100-101.

6. Aggarwal A, Jindal P (2014) Interaction of terbinafine hydrochloride with nicotinamide in solution and solid state. J Pharm Investig 44: 23-32.

7. Cevc G, Vierl U (2010) Nanotechnology and the transdermal route: A state of the art review and critical appraisal. J Control Release 141: 277-299.

8. Alberti I, Kalia YN, Naik A, Bonny JD, Guy RH (2001) Effect of ethano and isopropyl myristate on the availability of topical terbinafine in human stratum corneum, in vivo. Int J Pharm 219: 11-19.
9. Güngör S, Erdal MS, Aksu B (2013) New formulation strategies in topical antifungal therapy. J Chem Dermatol Sci Appl 3: 56.

10. Ruben MJ, Luis EJ, Alejandra C, Katherine H, Juan BK, et al. (2005) The bactericidal effect of silver nanoparticles. Nanotechnology 16: 2346.

11. Panacek A, Kvítek L, Prucek R, Kolar M, Večeřová R, et al. (2006) Silver colloid nanoparticles: Synthesis, characterization, and their antibacterial activity. J Phys Chem A 110: 16248-16253.

12. Panáček A, Kolář M, Večeřová R, Prucek R, Soukupová J, et al. (2009) Antifungal activity of silver nanoparticles against Candida spp. Biomaterials 30: 6333-6340.

13. Siddhartha S, Tanmay B, Arnab R, Gajendra S, Ramachandrarao P, et al. (2007) Characterization of enhanced antibacterial effects of novel silver nanoparticles. Nanotechnology 18: 225103.

14. Karri VVSN, Raman S, Kuppusamy G, Mulukutla S, Ramaswamy S, et al. (2015) Terbinafine hydrochloride loaded nanoemulsion based gel for topical application. J Pharm Investig 45: 79-89.

15. Karri VVSN, Raman S, Elango H, Venkata S, Yamjala K, et al. (2014) Development and validation of RP-UFLC method for the estimation of Terbinafine $\mathrm{Hcl}$ in pharmaceutical Nanoemulsion gel. Int J Pharm Pharm Sci 6: 586-590.

16. Franz TJ (1975) Percutaneous absorption. on the relevance of in vitro data. J Investig Dermatol 64: 190-195.

17. Salunkhe SS, Bhatia NM, Pokharkar VB, Thorat JD, Bhatia MS (2013) Topical delivery of Idebenone using nanostructured lipid carriers: evaluations of sun-protection and anti-oxidant effects. J Pharm Investig 43: 287-303.

18. Barot BS, Parejiya PB, Patel HK, Gohel MC, Shelat PK (2012) Microemulsion-based gel of terbinafine for the treatment of onychomycosis: Optimization of formulation using D-optimal design. AAPS Pharm Sci Tech 13: 184-192.

19. Zhu W, Guo C, Yu A, Gao Y, Cao F, et al. (2009) Microemulsion-based hydrogel formulation of penciclovir for topical delivery. Int J Pharm 378: 152-158

20. Arumugam N, Kandasamy S, Sekar M (2012) In-vitro antifungal activity of Hybanthus enneaspermus F Muell. Int J Pharm Pharm Sci 4: 594-596.

21. Rao S, Barot T, Rajesh KS, Jha L (2015) Formulation, optimization and evaluation of microemulsion based gel of Butenafine Hydrochloride for topical delivery by using simplex lattice mixture design. J Pharm Investig pp: 1-12.

22. Alvarez-Román R, Naik A, Kalia YN, Guy RH, Fessi H (2004) Enhancement of topical delivery from biodegradable nanoparticles. Pharm Res 21: 1818-1825.

23. Peltola S, Saarinen-Savolainen P, Kiesvaara J, Suhonen TM, Urtti A (2003) Microemulsions for topical delivery of estradiol. Int J Pharm 254: 99-107.

24. Trotta M (1999) Influence of phase transformation on indomethacin release from microemulsions. J Control Release 60: 399-405. 